\title{
Let's Not Miss the Forest for the Trees: A Reply to Montefinese and Vinson's (2015) Commentary on Vieth et al. (2014)
}

\author{
Harrison E. Vieth ${ }^{1 *}$, Katie L. McMahon ${ }^{2}$ and Greig I. de Zubicaray ${ }^{3}$ \\ ${ }^{1}$ School of Psychology, The University of Queensland, Brisbane, QLD, Australia, ${ }^{2}$ Centre for Advanced Imaging, The \\ University of Queensland, Brisbane, QLD, Australia, ${ }^{3}$ Faculty of Health, Institute of Health and Biomedical Innovation, \\ Queensland University of Technology, Brisbane, QLD, Australia
}

Keywords: lexical access, competition, semantic interference, picture naming, shared features, distinctive features

\section{OPEN ACCESS}

Edited by:

F-Xavier Alario,

CNRS and Aix-Marseille Université,

France

Reviewed by:

Francesca Peressotti,

University of Padova, Italy

*Correspondence:

Harrison E. Vieth

harrisonvth@gmail.com

Specialty section:

This article was submitted to

Language Sciences,

a section of the journal

Frontiers in Psychology

Received: 15 July 2015 Accepted: 12 December 2015

Published: 06 January 2016

Citation:

Vieth HE, McMahon KL and de Zubicaray Gl (2016) Let's Not Miss the

Forest for the Trees: A Reply to

Montefinese and Vinson's (2015) Commentary on Vieth et al. (2014).

Front. Psychol. 6:1984.

doi: 10.3389/fpsyg.2015.01984
In Vieth et al. (2014a), we conducted three experiments to examine semantic relatedness effects in the picture-word interference (PWI) paradigm. According to the lexical selection by competition account of spoken word production, feature overlap between the target picture and related distractor word induces semantic interference. However, this account has been challenged by studies demonstrating semantic facilitation in the PWI paradigm (e.g., Costa et al., 2005; Mahon et al., 2007; but see Hutson and Damian, 2014; Sailor and Brooks, 2014; Vieth et al., 2014b). In Vieth et al. (2014a), we investigated whether some reports of semantic facilitation in PWI might be due to the influence of distinctive features, i.e., features that quickly distinguish a concept from other similar concepts, as previous studies had not controlled for this variable (e.g., Costa et al., 2005; Mahon et al., 2007; Sailor and Brooks, 2014). In Experiment 3, we observed semantic interference for distractor words denoting a non-distinctive feature (e.g., knee) visible in the target picture (e.g., CAMEL), but no interference for distractor words denoting a distinctive feature (e.g., hump) compared to matched sets of distractors denoting unrelated features. We argued this finding is consistent with lexical selection by competition accounts, and might entail additional spread of activation to related concepts that share the non-distinctive feature via the appropriate category node (e.g., Animals). In their commentary, Montefinese and Vinson (2015) arrive at the opposite conclusion, arguing that feature distinctiveness does not affect the degree of interference in PWI. Here, we respond to each of their objections.

Montefinese and Vinson's (2015) principal objection concerned the statistical significance tests of the higher order interaction between distractor relatedness, distinctiveness and stimulus onset asynchrony (SOA), and partial interaction of relatedness and distinctiveness at the $-150 \mathrm{~ms}$ SOA. Our response to this objection revisits longstanding debates in experimental psychology about the use of arbitrary alpha levels to justify dichotomous decisions and failure to consider the size of effects (e.g., Rosnow and Rosenthal, 1989; Kirk, 2003; Cumming, 2014). In Vieth et al. (2014a), the $p$-values for the higher-order interaction by participants and by items were 0.06 and 0.078 , respectively, and the interaction was a medium sized effect (partial $\eta^{2}=0.10$ and 0.11 , respectively; e.g., Vacha-Haase and Thompson, 2004). Montefinese and Vinson misrepresent the two-way interaction as being "far from significant." In fact, this partial interaction was statistically significant in the items analysis and a larger effect $\left(p=0.023\right.$; partial $\left.\eta^{2}=0.21\right)$. Surprisingly, Montefinese and Vinson chose to cite Nieuwenhuis et al. (2011) to support their criticism of the statistical significance tests of the interactions, yet those authors wrote: "as famously noted by Rosnow and Rosenthal, 'surely, God loves the 0.06 nearly as much as the 0.05.' Thus, when making a comparison between two effects, researchers should report the statistical significance 
of their difference rather than the difference between their significance levels." (p. 1105). This is precisely the approach taken in Vieth et al. (2014a): interactions were reported and an arbitrary 0.05 level was not used to justify a dichotomous decision (cf. Montefinese and Vinson, 2015).

Of note, other researchers have followed marginally significant interactions with planned comparisons in PWI studies and offered discussions about why higher order interactions are particularly difficult to detect (e.g., Starreveld and La Heij, 1995; Damian and Martin, 1999). This two-pronged approach is preferred because semantic relatedness effects (i.e., interference and facilitation) in PWI are defined and demonstrated by planned comparisons of related vs. unrelated distractors. Costa et al. (2005) reported facilitation with related feature distractors using this approach. In Vieth et al. (2014b) we likewise contrasted distinctive and non-distinctive feature distractors with their respective matched unrelated distractor sets, as these were the chief comparisons we were interested in examining prior to commencing data collection. We were unable to create perfectly matched sets of distinctive and non-distinctive distractors, as we noted. Hence, our hypotheses applied to only a subset of the data contributing to the interaction term, as there was no a priori reason for contrasting the two unrelated distractor sets. Only non-distinctive feature distractors showed a significant interference effect both by participants $(p=0.011)$ and by items $(p=0.003)$, of moderate to large effect size $(d=$ 0.52 and 0.69 , respectively). It is worth emphasizing planned contrasts are typically conducted whether or not the overall Ftest is significant; at least this is the convention according to introductory experimental design and statistics textbooks (e.g., Kirk, 1982; Rosenthal and Rosnow, 1985; Keppel, 1991; Lomax and Hahs-Vaughn, 2012).

Montefinese and Vinson (2015) next expressed concern about Vieth et al.'s failure to match distinctive and non-distinctive features across distractor sets in terms of a "crucial semantic variable" called dominance-or production frequency, noting that many of the non-distinctive distractors (e.g., bone, skin) were not listed in the McRae et al.'s (2005) feature production norms. Production frequency refers to the number of participants that list a particular feature for a given concept in feature norming tasks, and has been interpreted as a measure of semantic saliency (e.g., Cree et al., 2006). Montefinese and Vinson argued that as many of the non-distinctive distractors were not listed in the McRae et al. norms, they were not salient for their related target concepts, and therefore were not sufficiently strong lexical competitors to induce the significant interference effect that we observed. As this argument relies on the novel assumption that dominance is a crucial measure of the strength of conceptual feature activation in PWI, it is worth examining in more detail.

For pragmatic purposes, researchers impose limits on both the time allowed and on the number of features to be produced for a given concept in feature norming studies, and then only include features in the resulting norms if a minimum number of participants listed them (e.g., 5/30 in McRae et al.'s, 2005). It is not surprising then, that feature norms do not represent exhaustive listings for each concept. Indeed as Montefinese et al. (2014) put it: "in a (theoretically) ideal experiment, if we asked a group of participants to list all of the possible features of a given concept, without posing any temporal or quantitative limits, we would end having a very large number of features with a dominance value close or equal to 1 . In fact, even the less important feature for that concept would eventually be listed, by most of the participants, making dominance a poor measure of a feature's importance/salience for a concept representation." (italics added; p. 356-357). We concur with Montefinese et al. (2014) view.

As Montefinese and Vinson (2015) noted, we were careful to match our PWI stimuli on a range of variables. In particular, we ensured associative relations between distractors and targets were minimal (Nelson et al., 2004). In free-association tasks, participants are given a cue word and required to list words that come to mind, a substantial proportion of which are features (De Deyne and Storms, 2008). Thus, "free association probabilities index the relative accessibility of related words in memory" (Nelson et al., 2004, p. 402). It should be apparent that dominance and free association probability are both measures of the relative availability of lexical knowledge. The potential collinearity between the two measures was acknowledged by Montefinese et al. (2014): "Consequently, if we consider the feature listing as a type of controlled word association task wherein the words are restricted to the features (Cree et al., 2006), we could presume that the order of production would also capture the lexical association link between concept and feature." (pp. 366-367). It therefore should not come as a surprise that the majority of our distractor stimuli in Vieth et al. (2014a) had low dominance, because features with high free association probabilities/high production frequencies had been mostly eliminated during their construction. However, the correlation is not perfect. When presented with the cue CAMEL, only a small proportion $(22 / 148$ or $15 \%)$ of the participants in the Nelson et al. (2004) normative study freely associated the concept with the feature hump, indicating it was not particularly accessible or salient to them. As Montefinese and Vinson point out, a higher proportion listed hump in McRae et al. (2005) normative study due to the use of an explicit instruction to list only the features of a CAMEL. Of the two measures, our view is that lexical association is the more important variable to control, as it has already been shown to influence naming latencies in PWI experiments.

Further, there is no evidence to support Montefinese and Vinson's claim of "substantial differences" in dominance of the distinctive and non-distinctive features in Vieth et al. The median production frequency for our distinctive distractors was low, only $8 / 30$ (or 27\%) according to McRae et al.'s (2005) norms. While dominance has been shown to influence decision latencies on feature verification tasks, the classic studies showing these effects contrasted high (i.e., greater than 50\%) vs. low production frequencies (e.g., Conrad, 1972; Ashcraft, 1978). Feature verification, in which participants are asked to indicate whether given features are true of target concepts (e.g., by reading property statements), also differs considerably from instructing participants to name a target picture while ignoring a distractor word. This difference in procedures is important to emphasize, as it is well-established that semantic relatedness effects depend 
critically on the nature of the experimental task. Given all of the above, we find little reason to entertain Montefinese and Vinson's conjecture about dominance significantly influencing our findings.

Aside from dominance, another theoretical issue introduced by Montefinese and Vinson (2015) in their commentary is the level-of-specificity between distractor and target in PWI. For example, (Hantsch et al., 2005; also Hantsch and Mädebach, 2013) reported that subordinate level distractors (e.g., Mini) produced interference when related objects were named at the basic level (e.g., CAR). Montefinese and Vinson (2015) speculate our findings might be akin to a level-ofspecificity effect and propose that the presence of the feature denoted by the distractor in the target picture (e.g., knee) might "permit further activation of its name as a potentially plausible alternative to the basic-level target name" (e.g., CAMEL).

Montefinese and Vinson do not provide an adequate explanation of the mechanism by which merely raising the lexical activation level of a feature-level distractor representation would make it a plausible response when the instruction is to name the entire target object (e.g., in Hantsch et al.'s level-of-specificity manipulation above, Mini and CAR are appropriate names for a picture of a Mini as they both denote the entire object, unlike wheel). We note the similarity of this proposal with Mahon et al.'s (2007) response exclusion account that attributes semantic interference to a post-lexical decision mechanism operating according to response-relevant criteria. As we acknowledged in Vieth et al. (2014b), the response exclusion account could possibly be modified to explain our finding by assuming visible features of target pictures constitute response relevant criteria, despite the instruction to name the whole object. However, this would involve abandoning Mahon et al.'s (2007) assumption that conceptual feature overlap does not constitute a responserelevant criterion.

Montefinese and Vinson's (2015) more empirical objection to our finding of semantic interference concerned the $-150 \mathrm{~ms}$ SOA at which the significant effect was observed in Experiment 3. Specifically, they characterized the result as "temporallyselective," implying the effect should have been observed at both 0 and -150 ms SOAs in the one experiment. In PWI timecourse studies, the semantic interference effect for category coordinates is typically found at either -150 or $0 \mathrm{~ms}$ SOAs, yet the literature shows the effect is often not significant at both of these SOAs within the same experiment (e.g., La Heij et al., 1990; Schriefers et al., 1990; Starreveld and La Heij, 1996; Damian and Martin, 1999; Damian and Bowers, 2003). In Sailor and Brooks' (2014) PWI study, significant interference from feature distractors was

\section{REFERENCES}

Ashcraft, M. H. (1978). Property dominance and typicality effects in property statement verification. J. Verbal Learn. Verbal Behav. 17, 155-164. doi: 10.1016/S0022-5371(78)90119-6

Conrad, C. (1972). Cognitive economy in semantic memory. J. Exp. Psychol. 92, 149-154. doi: 10.1037/h0032072 observed at the $0 \mathrm{~ms}$ SOA but not at $-150 \mathrm{~ms}$. This variability in the reported results for early and $0 \mathrm{~ms}$ SOAs likely reflects procedural differences across PWI studies, such as the use of written vs. auditory distractors, or central vs. random positioning of written distractors. In the PWI procedure, auditory distractors take longer to process than written ones, so an interference effect is more likely to be obtained at earlier SOAs (see Damian and Martin, 1999). Less-predictable placement of distractor words will likewise affect processing time. Consequently, there is no empirical reason to accept Montefinese and Vinson's stipulation that significant interference for related feature distractors should occur at both SOAs in the one PWI experiment.

According to the lexical selection by competition account, semantic interference is the result of a tradeoff between the priming of the target representation by the distractor and the priming of the distractor's lexical representation by the target (e.g., Roelofs, 1992). Montefinese and Vinson (2015) objected to our adopting this interpretation for the significant interference effect at $-150 \mathrm{~ms}$ (e.g., Roelofs, 1992; Starreveld and La Heij, 1996). Their premise is that activation cannot spread from target to distractor at the $-150 \mathrm{~ms}$ SOA as the target picture is yet to be presented. Logically, this criticism would also apply to the reported findings of semantic interference for category-coordinates at $-150 \mathrm{~ms}$ and earlier SOAs, and so challenge lexical-selection-by competition accounts, if the premise were accurate (e.g., La Heij et al., 1990; Schriefers et al., 1990; Starreveld and La Heij, 1995, 1996; Damian and Martin, 1999). However, Montefinese and Vinson's (2015) premise reflects a misunderstanding of the typical PWI procedure that involves written distractor words remaining on screen while the target picture is presented and until the participant responds (e.g., Vieth et al., 2014a). As naming latencies in PWI are usually between 600 to $800 \mathrm{~ms}$, this provides ample time for target-to-distractor priming.

In conclusion, we find none of the theoretical, empirical, or statistical objections raised by Montefinese and Vinson (2015) to be particularly compelling, as they reflect a combination of conjecture, misunderstanding and misrepresentation. However, we concur with them that the details of conceptual representations remain underspecified in production models. To this end, further empirical work is needed, rather than commentary.

\section{FUNDING}

This work was supported by Australian Research Council Discovery Project Grants DP1092619 and DP150103997. the semantic interference effect in the picture-word interference paradigm. Psychon. Bull. Rev. 12, 125-131. doi: 10.3758/BF03196357

Cree, G. S., McNorgan, C., and McRae, K. (2006). Distinctive features hold a privileged status in the computation of word meaning: implications for theories of semantic memory. J. Exp. Psychol. Learn. Mem. Cogn. 32, 643-658. doi: 10.1037/0278-7393.32.4.643 
Cumming, G. (2014). The new statistics: why and how. Psychol. Sci. 25, 7-29. doi: $10.1177 / 0956797613504966$

Damian, M., and Bowers, J. (2003). Locus of semantic interference in picture-word interference tasks. Psychon. Bull. Rev. 10, 111-117. doi: 10.3758/BF03196474

Damian, M. F., and Martin, R. C. (1999). Semantic and phonological codes interact in single word production. J. Exp. Psychol. Learn. Mem. Cogn. 25, 345-361. doi: 10.1037/0278-7393.25.2.345

De Deyne, S., and Storms, G. (2008). Word associations: network and semantic properties.Behav. Res. Methods 40, 213-231. doi: 10.3758/BRM.40.1.213

Hantsch, A., Jescheniak, J. D., and Schriefers, H. (2005). Semantic competition between hierarchically related words during speech planning. Mem. Cogn. 33, 984-1000. doi: 10.3758/BF03193207

Hantsch, A., and Mädebach, A. (2013). What does the articulatory output buffer know about alternative picture names? Evidence against the response-exclusion hypothesis. Lang. Cogn. Process. 28, 684-700. doi: 10.1080/01690965.2011.595725

Hutson, J., and Damian, M. F. (2014). Semantic gradients in picture-word interference tasks: is the size of interference effects affected by the degree of semantic overlap? Front. Psychol. 5:872. doi: 10.3389/fpsyg.2014.00872

Keppel, G. (1991). Design and Analysis: A Researcher's Handbook, 3rd Edn. Englewood Cliffs, NJ: Prentice Hall.

Kirk, R. E. (1982). Experimental Design: Procedures for the Behavioral Sciences, 2nd Edn. Belmont, CA: Brooks/Cole.

Kirk, R. E. (2003). “The Importance of Effect Magnitude," in Handbook of Research Methods in Experimental Psychology, eds S. F. Davis (Malden, MA: Blackwell), 83-105.

La Heij, W., Dirkx, J., and Kramer, P. (1990). Categorical interference and associative priming in picture naming. Br. J. Psychol. 81, 511-525. doi: 10.1111/j.2044-8295.1990.tb02376.x

Lomax, R. G., and Hahs-Vaughn, D. L. (2012). An Introduction to Statistical Concepts for Education and Behavioral Sciences, 3rd Edn. New York, NY: Routledge.

Mahon, B., Costa, A., Peterson, R., Vargas, K., and Caramazza, A. (2007). Lexical selection is not by competition: a reinterpretation of semantic interference and facilitation effects in the picture-word interference paradigm. J. Exp. Psychol. Learn. Mem. Cogn. 33, 503-535. doi: 10.1037/0278-7393.33.3.503

McRae, K., Cree, G. S., Seidenberg, M. S., and McNorgan, C. (2005). Semantic feature production norms for a large set of living and nonliving things. Behav. Res. Methods 37, 547-559. doi: 10.3758/BF03192726

Montefinese, M., Ambrosini, E., Fairfield, B., and Mammarella, N. (2014). Semantic significance: a new measure of feature salience. Mem. Cogn. 42, 355-369. doi: 10.3758/s13421-013-0365-y

Montefinese, M., and Vinson, D. (2015). Can the humped animal's knee conceal its name? commentary on: "the roles of shared vs. distinctive conceptual features in lexical access." Front. Psychol. 6:418. doi: 10.3389/fpsyg.2015.00418
Nelson, D. L., McEvoy, C. L., and Schreiber, T. A. (2004). The university of south florida free association, rhyme, and word fragment norms. Behav. Res. Methods Instrum. Comput. 36, 402-407. doi: 10.3758/BF03195588

Nieuwenhuis, S., Forstmann, B. U., and Wagenmakers, E. J. (2011). Erroneous analyses of interactions in neuroscience: a problem of significance. Nat. Neurosci. 14, 1105-1107. doi: 10.1038/nn.2886

Roelofs, A. (1992). A spreading-activation theory of lemma retrieval in speaking. Cognition 42, 107-142. doi: 10.1016/0010-0277(92)90041-F

Rosenthal, R., and Rosnow, R. L. (1985). Contrast Analysis: Focused Comparisons in the Analysis of Variance. Cambridge: Cambridge University Press.

Rosnow, R. L., and Rosenthal, R. (1989). Statistical procedures and the justification of knowledge in psychological science. Am. Psychol. 44, 1276-1284. doi: 10.1037/0003-066X.44.10.1276

Sailor, K., and Brooks, P. J. (2014). Do part-whole relations produce facilitation in the picture-word interference task? Q. J. Exp. Psychol. 67, 1768-1785. doi: 10.1080/17470218.2013.870589

Schriefers, H., Meyer, A. S., and Levelt, W. J. M. (1990). Exploring the time course of lexical access in language production-picture-word interference studies. J. Mem. Lang. 29, 86-102. doi: 10.1016/0749-596X(90)90011-N

Starreveld, P. A., and La Heij, W. (1995). Semantic interference, orthographic facilitation, and their interaction in naming tasks. J. Exp. Psychol. Learn. Mem. Cogn. 21:686. doi: 10.1037/0278-7393.21.3.686

Starreveld, P. A., and La Heij, W. (1996). Time-course analysis of semantic and orthographic context effects in picture naming. J. Exp. Psychol. Learn. Mem. Cogn. 22, 896-918. doi: 10.1037/0278-7393.22.4.896

Vacha-Haase, T., and Thompson, B. (2004). How to estimate and interpret various effect sizes. J. Couns. Psychol. 51, 473-481. doi: 10.1037/0022-0167.51. 4.473

Vieth, H. E., McMahon, K. L., and de Zubicaray, G. I. (2014b). Feature overlap slows lexical selection: evidence from the picture-word interference paradigm. Q. J. Exp. Psychol. 67, 2325-2339. doi: 10.1080/17470218.2014.923922

Vieth, H., McMahon, K., and de Zubicaray, G. I. (2014a). The roles of shared vs. distinctive conceptual features in lexical access. Front. Psychol. 5:1014. doi: 10.3389/fpsyg.2014.01014

Conflict of Interest Statement: The authors declare that the research was conducted in the absence of any commercial or financial relationships that could be construed as a potential conflict of interest.

Copyright (c) 2016 Vieth, McMahon and de Zubicaray. This is an open-access article distributed under the terms of the Creative Commons Attribution License (CC BY). The use, distribution or reproduction in other forums is permitted, provided the original author(s) or licensor are credited and that the original publication in this journal is cited, in accordance with accepted academic practice. No use, distribution or reproduction is permitted which does not comply with these terms. 